\title{
Association between CBR1 polymorphisms and NSCLC in the Chinese population
}

\author{
YONG GUO $^{1}$, YINGYING SHEN ${ }^{1}$, YONGMING XIA ${ }^{2}$ and JIANZHONG GU ${ }^{1}$ \\ ${ }^{1}$ Department of Oncology, The First Affiliated Hospital of Zhejiang Chinese Medical University, Hangzhou, \\ Zhejiang 310006; ${ }^{2}$ Department of Oncology, Yuyao People's Hospital of Zhejiang, Yuyao, Zhejiang 315400, P.R. China
}

Received August 7, 2015; Accepted January 12, 2017

DOI: $10.3892 / \mathrm{ol} .2017 .6926$

\begin{abstract}
Carbonyl reductase 1 (CBR1) is theorized to participate in various cellular processes, such as signal transduction, apoptosis, carcinogenesis and drug resistance, and is highly expressed in certain malignancies, including lung tumors. Several studies have provided evidence that gene polymorphisms may affect susceptibility to non-small cell lung cancer (NSCLC). The present study aimed to investigate the association between the CBR1 single-nucleotide polymorphisms (SNPs) rs3787728 and rs2835267, and NSCLC in a Chinese population. The data indicated that the allele frequency in CBR1 rs3787728 was significantly different between patients with NSCLC and the controls [odds ratio $(\mathrm{OR})=1.209 ; 95 \%$ confidence interval $(\mathrm{CI})=1.013-1.442 ; \mathrm{P}=0.0349]$, and was significantly different between male patients with NSCLC and the corresponding controls $(\mathrm{OR}=1.278 ; 95 \% \mathrm{CI}=1.016-1.607$; $\mathrm{P}=0.0358)$. The CBR1 rs3787728 thymine $(\mathrm{T}) / \mathrm{T}$ allele homozygote was associated with an increased risk of NSCLC in all patients $(\mathrm{OR}=1.382 ; 95 \% \mathrm{CI}=1.019-1.875 ; \mathrm{P}=0.037)$, and patients possessing the rs $3787728 \mathrm{~T} / \mathrm{T}$ major allele homozygote exhibited a 1.537-fold greater risk with respect to developing lung squamous-cell carcinoma (SCC) in all patients $(95 \%$ $\mathrm{CI}=1.019-2.318 ; \mathrm{P}=0.0395)$. The CBR1 rs3787728 cytosine (C)/C allele homozygote was associated with a decreased risk of adenocarcinoma (ADC) in male patients ( $\mathrm{OR}=0.633 ; 95 \%$ $\mathrm{CI}=0.413-0.969$; $\mathrm{P}=0.0348)$; however, no significant association was observed in CBR1 rs2835267 between SNPs and SCC or ADC-type NSCLC. In conclusion, the results revealed that genetic polymorphisms of CBR1 rs3787728 were associated with susceptibility to NSCLC. Additional studies are required to identify the functional impact of CBR1 expression and activity in NSCLC.
\end{abstract}

Correspondence to: Mr. Jianzhong Gu, Department of Oncology, The First Affiliated Hospital of Zhejiang Chinese Medical University, 54 Youdian Road, Hangzhou, Zhejiang 310006, P.R. China E-mail: gjzddw@zcmu.edu.cn; gjzddw@163.com

Key words: Chinese population, non-small cell lung cancer, human carbonyl reductase 1 , polymorphism

\section{Introduction}

Lung cancer is a leading cause of cancer-associated mortality in the majority of countries (1), including China (2). Non-small cell lung cancer (NSCLC) constitutes $82 \%$ of all incidences of lung cancer (3). Pathologically, genetic and environmental interactions serve a key role in the development and progression of lung cancer (4). It is biologically possible that host genetic susceptibility is a factor in the development of lung cancer, and contributes to the variation in individual cancer risk (5).

Human carbonyl reductase 1 (CBR1) is ubiquitously expressed NADPH-dependent enzymes belonging to the short dehydrogenase/reductase (SDR) family, which is involved in catalyzing the conversion of various endogenous and xenobiotic carbonyl compounds to their respective alcohol derivatives (6). CBR1 is also hypothesized to participate in cellular processes such as signal transduction (7), apoptosis (8), carcinogenesis (9) and drug resistance (10), and serves an important role in endometrial cancer (11), acute myeloid leukemia (12) and hepatocellular carcinoma (13). Schlager and Powis (14) reported that the level of CBR1 activity was significantly increased in lung tumors compared within normal tissue. Numerous studies have provided evidence that gene polymorphisms may influence the susceptibility to NSCLC $(15,16)$. A study in the USA has identified that genetic polymorphisms of the CBR1 gene, rs3787728 and rs2835267, are associated with susceptibility to lung cancer (17). Although the USA and China are similar in certain ways, the living environment and ethnic background of the population is also different in some ways. In the present study, the association between rs3787728 and rs2835267, single-nucleotide polymorphisms (SNPs) of CBR1, and the NSCLC risk of the Chinese population was investigated, and the impact of these SNPs on susceptibility and clinicopathological characteristics of NSCLC was evaluated.

\section{Patients and methods}

Study population. A total of 500 patients with NSCLC and 500 unrelated but age-matched healthy controls were recruited from the Zhejiang Cancer Hospital (Hangzhou, China) between March 2011 and April 2012. All patients were of Han origin and lived roughly within the same geographic region in Zhejiang, China. The patients exhibited no history of previous primary cancer other than lung cancer. All patients 
were histologically diagnosed with NSCLC and any patients with a history of primary cancer, other than lung cancer. The control patients did not exhibit any lung-associated disease. A smoker was defined as a patient who had smoked $>10$ packs of cigarettes in their lifetime. A current smoker or former smoker was defined as a patient who was still smoking in the year or previous year of enrollment into the present study. Patients who had ever smoked but had not accumulated 1 pack/year were considered occasional smokers; these patients were treated as non-smokers in the present analysis. The Ethics Committee of the Zhejiang Cancer Hospital approved the present study, and all participants provided informed consent.

SNP selection and genotyping. CBR1 rs3787728 and rs 2835267 were selected according to a previous study (17). Genomic DNA was extracted from whole blood using the AxyPrep Blood Genomic DNA Miniprep kit (Axygen Scientific, Inc., Union City, CA, USA). The two CBR1 SNPs were then genotyped using Sequenom Mass Array matrix-assisted laser desorption ionization-time of flight mass spectrometry platform (Sequenom, San Diego, CA, USA). The polymerase chain reaction and single base extension primers were designed using Assay Design software version 3.0 (Sequenom) and synthesized by Sangon Biotech Co., Ltd. (Shanghai, China). The primers were as follows: rs3787728 forward, 5'-ACGTTG GATGATTTCCAGAGGATCCCTATC-3'; reverse, 5'-ACG TTGGATGTGACCTGCAGGATCCTGGTG-3'; extension, 5'-CCTTTTCCCTAAGTCGT-3'; rs2835267 forward, 5'-ACG TTGGATGACCTTTCAGTAGGGCTGTTC-3'; reverse, 5'-ACGTTGGATGCTAGCACTTGAGAATACCAG; extension, 5'-ACAGACACCAGAAAACAA-3'.

Statistical analysis. All statistical analyses were performed using SPSS 13.0 for Windows (SPSS, Inc., Chicago, IL, USA). Hardy-Weinberg equilibrium (HWE) testing was carried out for all SNPs using the $\chi^{2}$ test. $\mathrm{P}<0.001$ was considered to indicate a statistically significant difference. The $\chi^{2}$ test was used to assess the frequencies of the selected allele and genotype between the patients and the controls. The association between SNPs and the NSCLC risk was evaluated by computing the odds ratio (OR) and 95\% confidence interval (CI) from multivariate unconditional logistic regression analysis. All P-values were two-sided and $\mathrm{P}<0.05$ was considered to indicate a statistically significant difference.

\section{Results}

A total of 500 patients, consisting of 350 males and 150 females, and 500 healthy controls, consisting of 259 males and 240 females, with gender information for 1 control subject missed, were of Chinese Han origin. A total of 280 male and 21 female patients were smokers or former smokers, where as 189 male and 14 female controls were smokers or former smokers. A total of 1,000 participants, 331 with adenocarcinoma (ADC), 169 with squamous-cell carcinomas (SCC) and 500 healthy controls were successfully genotyped for rs3787728 and rs 2835267 polymorphisms of CBR1. The studied population was within HWE, $\mathrm{P}=0.86506$ for rs 3787728 and $\mathrm{P}=0.54904$ for rs 2835267 .

The association between allele frequency in rs 3787728 and rs2835267 CBR1 and the clinicopathological parameters and gender of patients with NSCLC was analyzed and is presented in Tables I-III. The allele frequency in rs3787728 was significantly different between the patients with NSCLC and the controls $(\mathrm{OR}=1.209 ; 95 \% \mathrm{CI}=1.013-1.442 ; \mathrm{P}=0.0349)$, and was also significantly different between male patients with NSCLC and male controls (OR=1.278; 95\% CI $=1.016-1.607$; $\mathrm{P}=0.0358)$. The thymine ( $\mathrm{T}$ ) allele in rs 3787728 was observed to be associated with an increased risk for NSCLC development, particularly for the development of NSCLC in males. With respect to allele frequencies in rs2835267, no statistical differences were observed between the case and control subjects $(\mathrm{P}=0.4595)$. With respect to allele frequencies in rs2835267, no statistical differences were observed between male NSCLCs and male controls $(\mathrm{P}=0.6842)$, between female NSCLCs and female controls ( $\mathrm{P}=0.4897)$, between patients with ADC and the controls $(\mathrm{P}=0.3566)$, or between patients with SCC and the controls $(\mathrm{P}=0.9059)$.

The association of the genotype frequency of rs 3787728 and rs2835267CBR1 with the clinicopathological parameters and gender of patients with NSCLC was analyzed and is presented in Tables IV-VI.Logistic regression analysis revealed that the rs3787728 $\mathrm{T} / \mathrm{T}$ allele homozygote was associated with an increased risk of NSCLC in all patients ( $\mathrm{OR}=1.382$; 95\% $\mathrm{CI}=1.019-1.875 ; \mathrm{P}=0.037)$, and patients exhibiting the rs3787728 T/T major allele homozygote exhibited a 1.537 -fold greater risk in developing lung SCC (95\% CI=1.019-2.318; $\mathrm{P}=0.0395)$. However, the rs3787728 cytosine (C)/C allele homozygote was associated with a decreased risk of ADC in male patients $(\mathrm{OR}=0.633 ; 95 \% \mathrm{CI}=0.413-0.969 ; \mathrm{P}=0.0348)$. There was no statistical difference with respect tothe CBR1 rs2835267 SNP genotype between the patients with NSLCLC and the controls, between patients with ADC and the controls, or between patients with SCC and the controls ( $\mathrm{P}>0.05)$. With respect to genotype frequencies in rs2835267, no statistical differences were observed between male NSCLCs and male controls ( $\mathrm{T} / \mathrm{T}$ vs. $\mathrm{C} / \mathrm{C}+\mathrm{C} / \mathrm{T}, \mathrm{P}=0.3768 ; \mathrm{C} / \mathrm{C}$ vs. $\mathrm{T} / \mathrm{T}+\mathrm{C} / \mathrm{T}$, $\mathrm{P}=0.5966)$ or between female NSCLCs and female controls ( $\mathrm{T} / \mathrm{T}$ vs. $\mathrm{C} / \mathrm{C}+\mathrm{C} / \mathrm{T}, \mathrm{P}=0.7844 ; \mathrm{C} / \mathrm{C}$ vs. $\mathrm{T} / \mathrm{T}+\mathrm{C} / \mathrm{T}, \mathrm{P}=0.3456$ ).

\section{Discussion}

The present study assessed the association between the CBR1 SNPs rs3787728 and rs2835267 and the risk of developing NSCLC, and identified that patients with the rs3787728 $\mathrm{T}$ allele exhibited an increased risk of developing NSCLC, particularly male patients with NSCLC. The CBR1 rs3787728 $\mathrm{T} / \mathrm{T}$ allele homozygote was associated with a higher risk for NSCLC and lung SCC in all patients, and the C/C allele homozygote was associated with a lower risk for ADC in male patients. However, the present study identified a lack of association between the polymorphisms of CBR1 rs2835267 with NSCLC, regardless of whether the NSCLC was of SCC or ADC type.

In humans, CBR1 is known as prostaglandin 9-ketoreductase (PG-9-KR) which inactivates prostaglandin $\mathrm{E} 2\left(\mathrm{PGE}_{2}\right)$ and other prostaglandins $(18,19)$. $\mathrm{PGE}_{2}$ serves an important role in inhibiting apoptosisand inducing angiogenesis (20). The inactivation of $\mathrm{PGE}_{2}$ by CBR1 may inhibit cancer angiogenesis (21), which serves an important role in the growth, progression and metastasis of cancer (22). A significant correlation between 


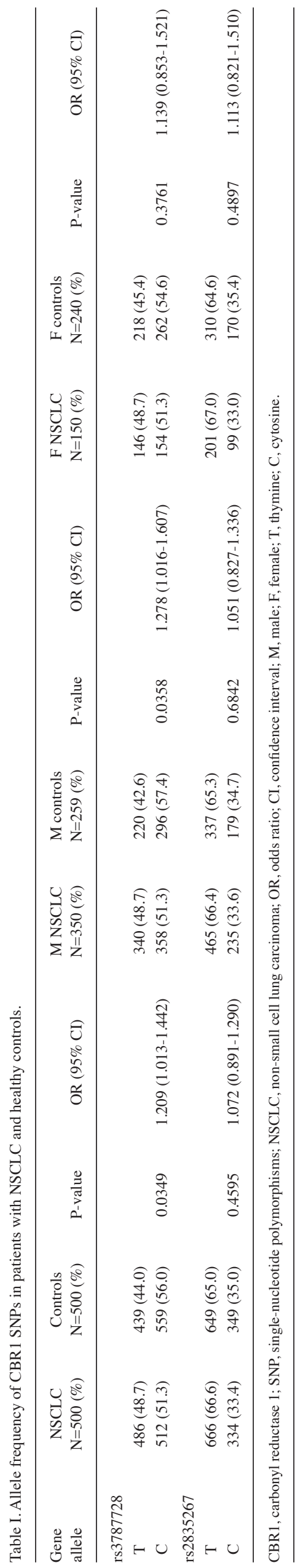

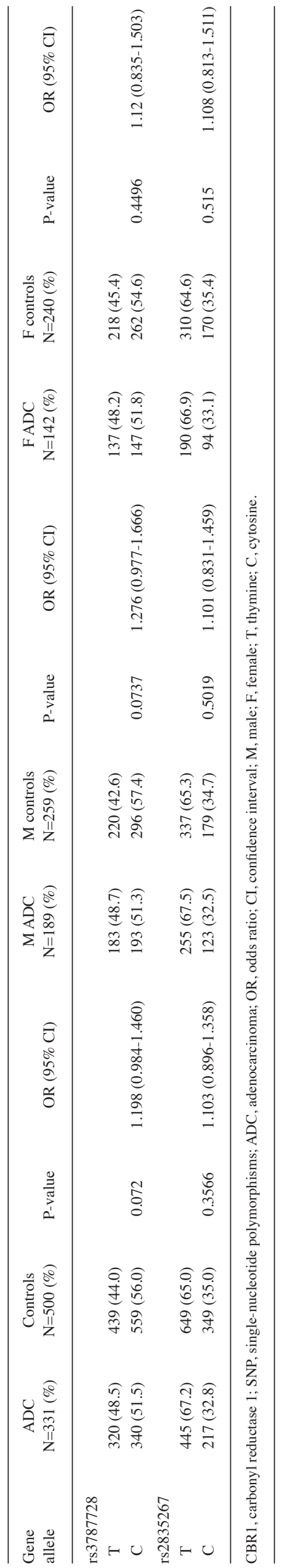




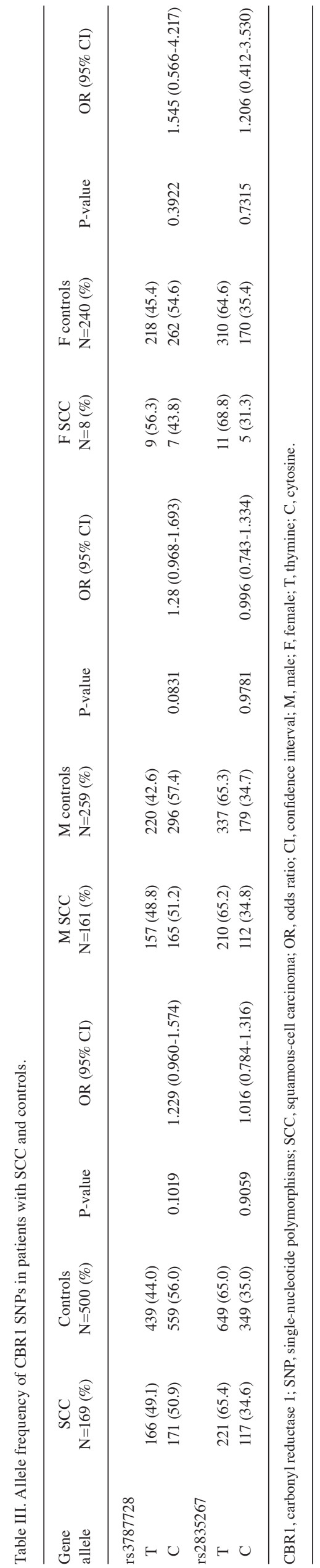

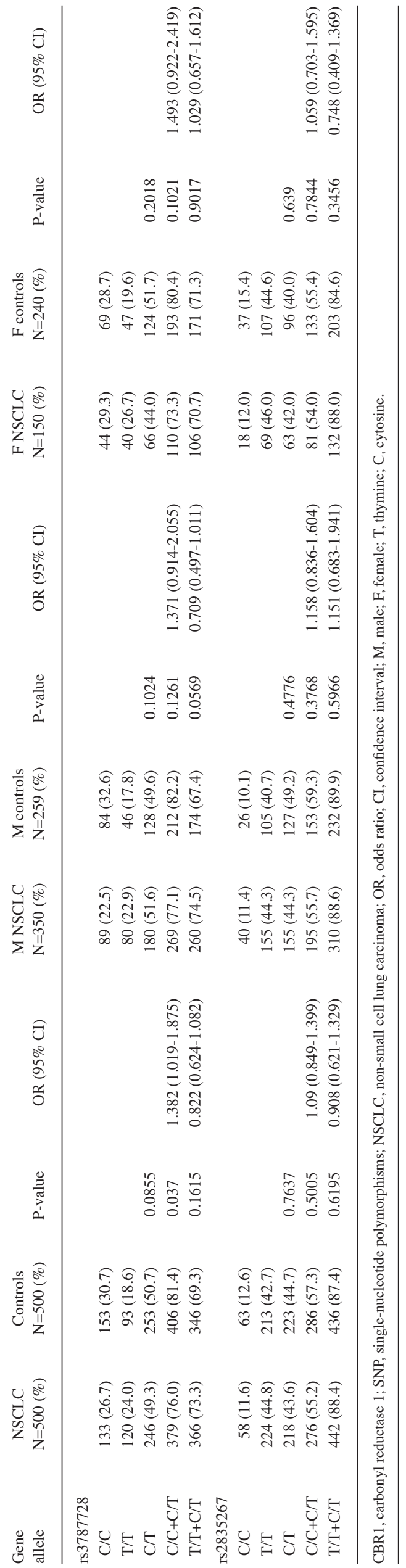



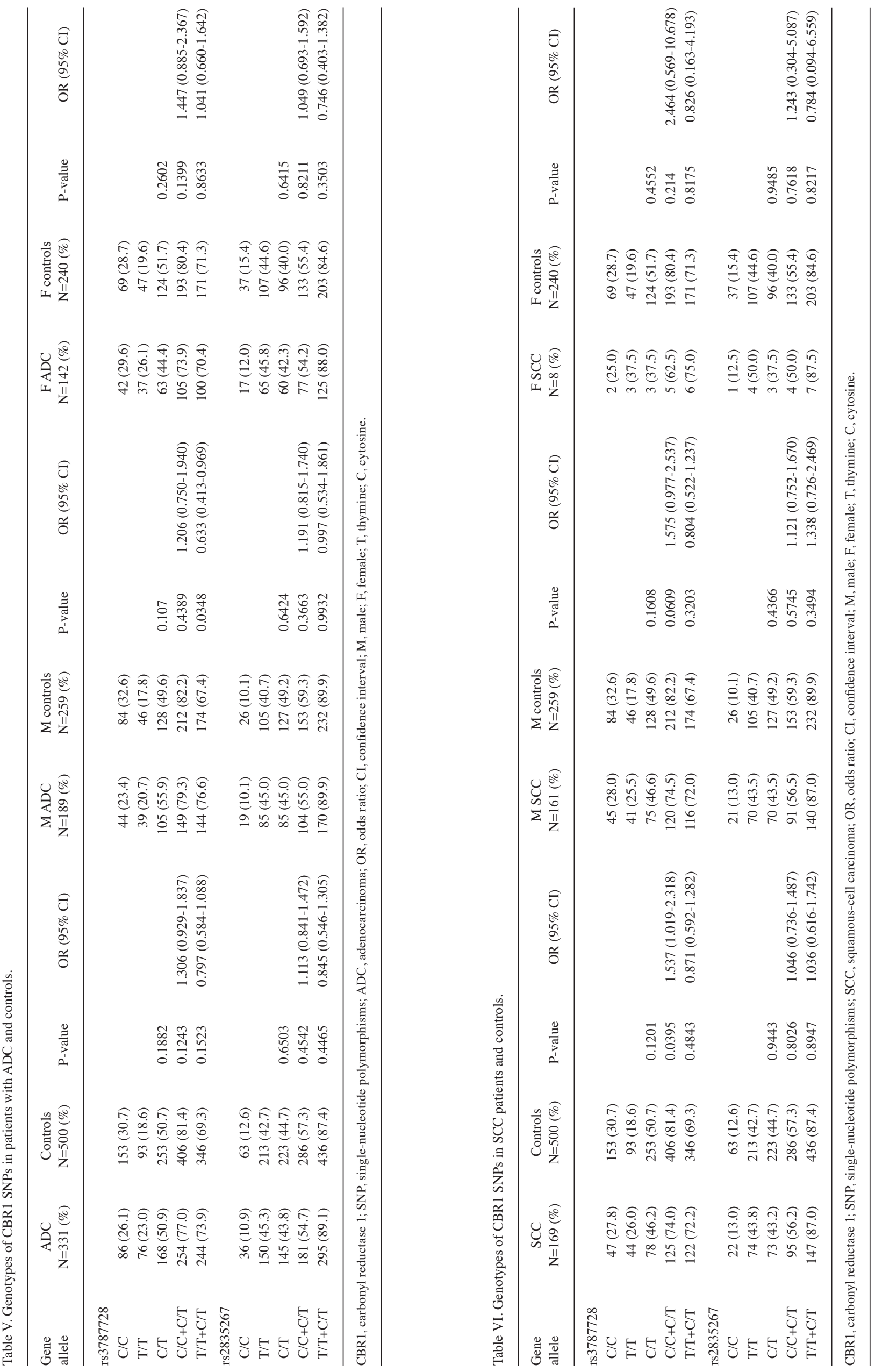
reduced CBR1 mRNA expression and active angiogenesis has been identified (21). Previous studies have revealed that CBR1 serves an important role in the metastasis of cancer cells by decreasing $\mathrm{PGE}_{2}$ levels $(19,21)$.

In addition, CBR1 reduces numerous xenobiotic carbonyl substrates to the corresponding alcohol metabolites (23). Analyses of the $>7,000$ compounds in cigarette smoke have identified $\sim 70$ carcinogens (24), the majority of which require metabolic activation to exert their cancer-causing effects (25). 4-methylnitrosamino-1-(3-pyridyl)-1-butanone (NNK) and benzo(a)pyrene $[\mathrm{B}(\mathrm{a}) \mathrm{P}]$ are xenobiotic substrates of CBR1 (23), which are present in tobacco and tobacco smoke $(26,27)$. NNK and $\mathrm{B}(\mathrm{a}) \mathrm{P}$ are theorized to serve important roles in human tobacco-associated cancer (25). CBR1 activity serves plays a role during the metabolism of NNK in human lungs (26). $\mathrm{B}(\mathrm{a}) \mathrm{P}$ may upregulate the expression of CBR1 in human lung tissues and lung cancer cells via the aryl hydrocarbon receptor pathway (23). Individual susceptibility to lung cancer may be affected by polymorphisms in genes such as CBR1, which encode proteins responsible for the detoxification of carcinogens in cigarette smoke (23). CBR1 polymorphisms may affect thebalance of activation vs. detoxification of NNK and $\mathrm{B}(\mathrm{a}) \mathrm{P}$, thus increasing vulnerability or protection to the lungs of smokers (23).

In accordance with the aforementioned previous studies, the present study indicated that CBR 1 may be a susceptibility gene involved in the development of NSCLC. In the present study, the individuals with CBR1 rs3787728 T/T homozygote exhibited a 1.382-fold increased risk for developing NSCLC and a 1.537 -fold increased risk for developing lung SCC, compared with the $\mathrm{C} / \mathrm{C}$ homozygote or $\mathrm{T} / \mathrm{C}$ heterozygote individuals. However, $\mathrm{C} / \mathrm{C}$ homozygote individuals exhibited a 0.633-fold decreased risk for developing lung ADC. Notably, the CBR1 rs2835267 polymorphism itself was not associated with the risk of developing NSCLC, contrary tothe results of study by Modesto et al (17). These differences may be attributed to the distinct genetic background and the different living environments of the Chinese population compared with other populations.

To conclude, the present study identified that CBR1 rs3787728 polymorphisms are significantly associated with NSCLC in Chinese people. However, the small sample size may be a limitation of the present study; in addition, population stratification may have led to a bias. Additional studies are required to elucidate the functional impact of CBR1 expression and activity in NSCLC.

\section{Acknowledgements}

The present study was sponsored by a grant from the Zhejiang Provincial Science and Technology Project (grant no. 2015C33172).

\section{References}

1. Edwards BK, Noone AM, Mariotto AB, Simard EP, Boscoe FP, Henley SJ, Jemal A, Cho H, Anderson RN, Kohler BA, et al: Annual report to the nation on the status of cancer, 1975-2010, featuring prevalence of comorbidity and impact on survival among persons with lung, colorectal, breast, or prostate cancer. Cancer 120: 1290-1314, 2014.
2. He J and Chen W: 2012 Chinese cancer registry annual report. Military Medicine Society Press, Beijing, 2012.

3. Pao W and Chmielecki J: Rational, biologically based treatment of EGFR-mutant non-small-cell lung cancer. Nat Rev Cancer 10: 760-774, 2010

4. Schwartz AG, Wenzlaff AS, Bock CH, Ruterbusch JJ, Chen W, Cote ML, Artis AS, Van Dyke AL, Land SJ, Harris CC, et al: Admixture mapping of lung cancer in 1812 African-Americans. Carcinogenesis 32: 312-317, 2011.

5. Marshall AL and Christiani DC: Genetic susceptibility to lung cancer-light at the end of the tunnel? Carcinogenesis 34: 487-502, 2013.

6. Oppermann U: Carbonyl reductases: The complex relationships of mammalian carbonyl- and quinone-reducing enzymes and their role in physiology. Annu Rev Pharmacol Toxicol 47: 293-322, 2007.

7. Forrest GL and Gonzalez B: Carbonyl reductase. Chem Biol Interact 129: 21-40, 2000.

8. Tanaka M, Bateman R, Rauh D, Vaisberg E, Ramachandani S, Zhang C, Hansen KC, Burlingame AL, Trautman JK, Shokat KM, et al: An unbiased cell morphology-based screen for new, biologically active small mol-ecules. PLoS Biol 3: e128, 2005.

9. Tak E, Lee S, Lee J, Rashid MA, Kim YW, Park JH, Park WS, Shokat KM, Ha J and Kim SS: Human carbonyl reductase 1 upregulated by hypoxia renders resistance to apoptosis in hepatocellular carcinoma cells. J Hepatol 54: 328-339, 2011.

10. Bains OS, Karkling MJ, Grigliatti TA, Reid RE and Riggs KW: Two nonsynonymous single nucleotide polymorphisms of human carbonyl reduct-ase 1 demonstrate reduced in vitro metabolism of daunorubicin and doxorubicin. Drug Metab Dispos 37: 1107-1114, 2009.

11. Murakami A, Yakabe K, Yoshidomi K, Sueoka K, Nawata S, Yokoyama Y, Tsuchida S, Al-Mulla F and Sugino N: Decreased carbonyl reductase 1 expression promotes malignant behaviours by induction of epithelialmesenchymal transition and its clinical significance. Cancer Lett 323: 69-76, 2012.

12. Varatharajan S, Abraham A, Zhang W, Shaji RV, Ahmed R, Abraham A, George B, Srivastava A, Chandy M, Mathews V, et al: Carbonyl reductase 1 expression influences daunorubicin metabolism in acute myeloid leukemia. Eur J Clin Pharmacol 68: 1577-1586, 2012.

13. Huang W, Ding L, Huang Q, Hu H, Liu S, Yang X, Hu X, Dang Y, Shen S, Li J, et al: Carbonyl reductase 1 as a novel target of (-)-epigallocatechin gallate against hepatocellular carcinoma. Hepatology 52: 703-714, 2010.

14. Schlager JJ and Powis G: Cytosolic NAD (P)H:(quinone-acceptor) oxidoreductase in human normal and tumor tissue: Effects of cigarettes moking and alcohol. Int J Cancer 45: 403-409, 1990.

15. Gu J, Shen Y and Zhang Y: Association between interleukin-4 polymorphisms and environment and non small cell lung cancer in Chinese population. J Cancer Res Ther 10 (Suppl): C135-C139, 2014.

16. Zhang Y, Hua S, Zhang A, Kong X, Jiang C, Deng D and Wenlong B: Association between polymorphisms in COMT, PLCH1 and CYP17A1 and non-small cell lung cancer risk in Chinese nonsmokers. Clin Lung Cancer 14: 45-49, 2013.

17. Modesto JL, Hull A, Angstadt AY, Berg A, Gallagher CJ, Lazarus $\mathrm{P}$ and Muscat JE: NNK reduction pathway gene polymorphisms and risk of lung cancer. Mol Carcinog 54 (Suppl 1): E94-E102, 2015.

18. Waclawik A, Jabbour HN, Blitek A and Ziecik AJ: Estradiol17beta, prostaglandin E2 (PGE2) and the PGE2 receptor are involved in PGE2 positive feedback loop in the porcine endometrium. Endocrinology 150: 3823-3832, 2009.

19. Yokoyama Y, Xin B, Shigeto T, Umemoto M, Kasai-Sakamoto A, Futagami M, Tsuchida S, Al-Mulla F and Mizunuma H: Clofibric acid, a peroxisome proliferator-activated receptor alpha ligand, inhibits growth of human ovarian cancer. Mol Cancer Ther 6: 1379-1386, 2007.

20. Jain S, Chakraborty G, Raja R, Kale S and Kundu GC: Prostaglandin E2 regulates tumor angiogenesis in prostate cancer. Cancer Res 68: 7750-7759, 2008.

21. Takenaka K, Ogawa E, Oyanagi H, Wada H and Tanaka F: Carbonyl reductase expression and its clinical significance in non-small-cell lung cancer. Cancer Epidemiol Biomarkers Prev 14: 1972-1975, 2005.

22. Makrilia N, Lappa T, Xyla V, Nikolaidis I and Syrigos K: The role of angiogenesis in solid tumours: An overview. Eur J Intern Med 20: 663-671, 2009. 
23. Kalabus JL, Cheng Q, Jamil RG, Schuetz EG and Blanco JG: Induction of carbonyl reductase 1 (CBR1) expression in human lung tissues and lung cancer cells by the cigarette smoke constituent benzo[a]pyrene. Toxicol Lett 211: 266-273, 2012.

24. American CancerSociety:Tobacco and cancer.http://www.cancer. org/cancer/cancercauses/tobaccocancer/questionsaboutsmokingtobaccoandhealth/. Accessed October 20, 2014.

25. Hockley SL, Arlt VM, Brewer D, Te Poele R, Workman P, Giddings I and Phillips DH: AHR- and DNA damage-mediated gene expression responses induced by benzo(a)pyrene in human cell lines. Chem Res Toxicol 20: 1797-1810, 2007.
26. Atalla A, Breyer-Pfaff $U$ and Maser E: Purification and characterization of oxidoreductases-catalyzing carbonyl reduction of the tobacco-specific nitrosamine 4-methylnitrosamino-1-(3-pyridyl)-1-butanone (NNK) in human liver cytosol. Xenobiotica 30: 755-769, 2000.

27. Breyer-Pfaff U, Martin HJ, Ernst $M$ and Maser E: Enantioselectivity of carbonyl reduction of 4-methylnitrosamino-1-(3-pyridyl)-1-butanone by tissue fractions from human and rat and by enzymes isolated from human liver. Drug Metab Dispos 32: 915-922, 2004. 